\title{
Thyroid Function Variability in a Cohort of Healthy Pregnant Women: Effect of BMI, Smoking and Iodized Salt Consumption
}

\author{
Inés Velasco*, **, Berta Soldevila ${ }^{2,4 * *}$, Raquel MartínezMondejar5, Carla Muñoz ${ }^{2,4}$, Laura Ferrer ${ }^{2,4}$, Martínez M $^{6}$, \\ Núria Gassol $^{5}$, Fernando Moreno ${ }^{2,7}$, MaríaLuisa Granada ${ }^{2,7}$, Eduardo GarcíaFuentes ${ }^{8}$ and Manel Puig Domingo ${ }^{2,4 *}$
}

${ }^{1}$ Pediatrics, Gynecology \& Obstetrics Unit, Riotinto Hospital, Spain

${ }^{2}$ Endocrine, Thyroid \& Obesity Research Group. Germans Trias i Pujol Research Institute, Spain

${ }^{3}$ Pediatrics, Gynecology \& Obstetrics Department, Autonomous University of Barcelona, Spain

${ }^{4}$ Endocrinology \& Nutrition Department, Germans Trias i Pujol University Hospital, Spain

${ }^{5}$ Gynecology \& Obstetrics Department, Germans Trias i Pujol University Hospital, Spain

${ }^{6}$ Pediatrics Department, Germans Trias i Pujol University Hospital, Spain

${ }^{7}$ Clinical Biochemistry Department, Germans Trias i Pujol University Hospital, Spain

${ }^{8}$ Department of Gastroenterology, Virgen de la Victoria University Hospital, Spain

**Both authors contributed equally to this manuscript as co-first authors.

*Corresponding author: Inés Velasco, Pediatrics, Endocrine, Gynecology \& Obstetrics Unit, Riotinto Hospital, Thyroid \& Obesity Research Group, Gynecology \& Obstetrics Department, Germans Trias i Pujol Research Institute, Autonomous University of Barcelona, Spain

Prof Manel Puig Domingo, MD, PhD, Endocrinology \& Nutrition Department, Germans Trias i Pujol University Hospital, and Germans Trias i Pujol Research Institute, Camí de les escoles, carretera de Can Ruti s/n 08916 Badalona, Spain. Tel: +(34) 934978860; E-mail: mpuigd@igtp.cat

\section{Abstract}

Introduction: It remains unclear to what extent intrinsic maternal characteristics, lifestyle or diet and dietary supplements contribute to explain the variability of maternal thyroid function. The aim of this study was to analyse the effect of age, parity, pregestational body mass index (BMI), beta human chorionic gonadotropin ( $\beta$-hCG), thyroid autoantibodies, smoking habit and use of vitamins/supplements on maternal thyroid function variability throughout pregnancy.

Methods: A prospective, observational study was carried out including 339 healthy pregnant women from their first to their third trimester. Clinical and biological variables were registered at each stage. Univariate correlation and multiple linear regression analysis for thyroid parameters were performed in the three trimesters.

Results: Thyrotropin (TSH) in the first trimester (1T) was dependent on free thyroxine (FT4), maternal age, $\beta$-hCG and smoking habit. FT4 levels in the 1T were significantly lower in obese women $(0.937 \pm 0.078 \mathrm{ng} / \mathrm{dl})$, compared with overweighed $(0.981 \pm 0.14 \mathrm{ng} / \mathrm{dl})$ and normal weight women $(0.989 \pm 0.98 \mathrm{ng} / \mathrm{dl})(\mathrm{p}=0.012)$. Multiple linear regression showed that FT4 in all trimesters is significantly dependent on pregestational BMI. Additionally, TSH and FT4 in the 1T were significantly related to TSH and FT4 levels in second and third trimesters, respectively. All studied factors influenced TSH with different degree all-over the pregnancy. Dietary supplements did not modify maternal thyroid function.

Conclusion: FT4 availability during the first half of the gestation can be hindered by maternal overweight/obesity. Beside age, obesity and smoking, TSH and FT4 in the $1 \mathrm{~T}$ are very important regarding thyroid function variability in further stages of the pregnancy. 


\section{Introduction}

Thyroid hormones are involved in many processes during intrauterine life such as somatic growth, metabolic regulation, and neurodevelopment [1]. Maternal thyroid function has also been related to obstetric outcomes in different studies [2,3]. The developing foetus is completely dependent on maternal supply of these hormones during the first half of the pregnancy [4]; therefore, an adequate maternal thyroid function becomes crucial to achieve a successful intrauterine development [5]. In order to identify maternal thyroid dysfunction at early stages of gestation, it is important to fully understand the physiological changes that happen to the thyroid gland in pregnant women as well as the potential factors that may modulate the concentration of thyroid hormones in maternal serum [6]. Once fecundation occurs, $\beta$-hCG increases rapidly in order to guarantee implantation and supporting embryonic growth [7]. The interpretation of thyroid parameters in pregnant women should take into account $\beta$-hCG action and thus gestational age and reference ranges need to be trimester-specific [8]. Beta-hCG and thyrotropin (TSH) relationship usually show an inverse correlation in a linear way [9]. However, more recently it has been demonstrated that the thyrotrophic effect of $\beta$-hCG can be modulated by individual factors such as maternal age [10], parity, body mass index (BMI) [11], thyroid autoimmunity [12] or even by the sex of the fetus [13]. Additionally, women with subclinical hypothyroidism show an abnormal response to thyroidal stimulation by $\beta$-hCG [14]. It is not well known what the relative importance of maternal personal conditions is such as weight, iodine-enriched multivitamin complexes intake $[15,16]$, smoking habit and lifestyle [17] in thyroid function throughout the pregnancy. The aim of our research is to study the potential contribution of different factors in maternal thyroid function variability in pregnant women without maternal or fetal risk factors, as well as to identify profiles of low availability of maternal thyroxine (T4).

\section{Material and Methods}

\section{Study subjects}

The study included 339 healthy pregnant women assisted at a maternal primary health care clinic, the ASSIR La Riera (Badalona, Spain), recruited at first trimester (1T) of pregnancy (before 10 weeks of amenorrhea) and followed up to the third trimester (3T). Exclusion criteria were based on the presence of pregestational maternal and/or foetal disorders that might represent an obstetric or perinatal risk, as well as pregnant women with a previously known history of thyroid dysfunction, those who were taking thyroid hormone or antithyroid drugs before pregnancy or those recently exposed to iodinated antiseptics or radiologic contrasts. Pregestational body mass index was considered as quantitative variable $\left(\mathrm{kg} / \mathrm{m}^{2}\right)$ and also as qualitative variable (BMI category) with

i) Normal weight $\left(\mathrm{BMI}<25 \mathrm{~kg} / \mathrm{m}^{2}\right)$ ii) Overweight (BMI $\left.25.0-29.9 \mathrm{~kg} / \mathrm{m}^{2}\right)$ and

iii) Obesity (BMI $\left.\geq 30.0 \mathrm{~kg} / \mathrm{m}^{2}\right)$.

Smoking habit was recorded as smokers, non-smokers, and stopped smokers (women who had quitted it when they became pregnant or less than a year before).

All women provided blood samples at the three trimesters of gestation. All the samples were stored at $-80 \stackrel{\circ}{\circ}$ until the analysis. Beta-HCG was performed in $1 \mathrm{~T}$ blood analysis, as routine clinical practice. The study design and research aims were approved by the Ethics Committee of the Germans Trias i Pujol University Hospital (HUGTiP) and written consent was obtained from all the participants.

\section{Laboratory procedures}

Serum measurements TSH were performed on the automated Abbott Architect TSH Ref. 7K62 (Abbott Diagnostic Division, Longford, Ireland). Free T4 (FT4) was measured by the Abbott Architect FT4 Ref. 7K65 (Abbott Diagnostic Division, Longford, Ireland). Total $\beta$-hCG was measured by the automated Abbott Architecht Re. 7 K78 (Abbott Diagnostic Division, Longford, Ireland). Serum anti-thyroid peroxidase antibodies (anti-TPOAbs) measurements were performed on the automated Abbott Architect Ref. 2K47 (Abbott Diagnostic Division, Longford, Ireland). Serum anti-thyroglobulin antibodies (anti-TgAbs) measurements were performed on the automated Abbott Architect Ref. 2K46 (Abbott Diagnostic Division, Longford, Ireland).

\section{Statistical analysis}

Normal distribution of quantitative variables was assessed by the Kolmogorov-Smirnov test. Variables were expressed as mean and standard deviation (SD) or as median and interquartile range (P25-75) as appropriate. Quantitative variables were shown as the mean \pm SD and qualitative variables as percentages. The contrast hypothesis for two samples was evaluated with the Student's t-test, and for more than two samples, with an analysis of variance (ANOVA) test. The chi-square test was applied in case of categorical variables. The correlation between variables was determined using the Spearman test, designing multiple linear regression models in those cases where it was desired to predict the variance adjusted for other variables, besides the main variable. In all cases, the rejection level for a null hypothesis was alpha below 0.05 . All data were analysed using SPSS 20.0 (IBM SPSS Statistics).

\section{Results}

\section{Clinical and demographic variables}

All participants were classified as healthy pregnant women after a general medical examination. Table 1 shows the clinical variables of the participants. There were significant differences in maternal age according to parity $(30.2 \pm 5.0$ years for parity 
0 ; $32.4 \pm 5.3$ years for parity $1 ; 33.6 \pm 4.7$ years for parity 2 and $33.0 \pm 5.0$ years for parity above $2 ; \mathrm{p}<0.001$ ) and level of education $(30.9 \pm 5.9$ years in low level; $31.8 \pm 5.1$ in middle level and $33.3 \pm 3.9$ years in high level of education; $p=0.016$ ). There were significant differences in smoking habit during pregnancy according to the level of education, $(36.2 \%$ of smokers in women with low level of education, $20.8 \%$ in middle level group and $6.5 \%$ in women with high level of education; $\mathrm{p}<0.001$ ).

Table 1: Demographic and clinical characteristics of participants.

\begin{tabular}{|c|c|c|}
\hline & Mean (SD) & N (\%) \\
\hline Maternal age (years) ${ }^{\mathrm{a}}$ & $31.9(5.2)$ & \\
\hline Maternal weight $(\mathrm{kg})^{\mathrm{a}}$ & $65.1(13.7)$ & \\
\hline Body mass index (BMI) ${ }^{a}$ & $24.8(4.9)$ & \\
\hline \multicolumn{3}{|c|}{ BMI Category } \\
\hline$<25$ & & $206(60.9)$ \\
\hline $25.0-29.9$ & & $80(23.7)$ \\
\hline$\geq 30$ & & $52(15.4)$ \\
\hline \multicolumn{3}{|c|}{ Parity } \\
\hline 0 & & $115(33.9)$ \\
\hline 1 & & $117(34.5)$ \\
\hline 2 & & $62(18.3)$ \\
\hline$>2$ & & $45(13.3)$ \\
\hline Gestational age (weeks) ${ }^{\mathrm{b}}$ & $7.6(1.5)$ & \\
\hline \multicolumn{3}{|c|}{ Level of Education } \\
\hline Low (none/primary) & & $94(27.7)$ \\
\hline Middle (secondary) & & $168(49.6)$ \\
\hline High (higher education) & & 77 (22.7) \\
\hline \multicolumn{3}{|c|}{ Smoking Habit } \\
\hline Nonsmokers & & $227(67)$ \\
\hline Stopped smokers & & $38(11.2)$ \\
\hline Smokers & & $74(21.8)$ \\
\hline
\end{tabular}

\begin{tabular}{|c|c|c|}
\hline \multicolumn{2}{|c|}{ WorkingWomen } \\
\hline No & & $63(18.6)$ \\
\hline Yes & & $276(81.4)$ \\
\hline Consumption of Iodised Salt & & $257(75.8)$ \\
\hline No & & $82(24.2)$ \\
\hline Yes & & \\
\hline \multicolumn{2}{|c|}{ Use of Supplements } \\
\hline None & & $113(33.3)$ \\
\hline Potassium iodide & & $104(30.7)$ \\
\hline Multivitamins & & $122(36)$ \\
\hline
\end{tabular}

${ }^{a}$ At the time of recruitment.

${ }^{\mathrm{b}}$ Adjusted by fetal ultrasonography at the first visit.

\section{Maternal thyroid function}

The maternal values of TSH and FT4 in the three trimesters of pregnancy are summarized in Tables $2 \& 3$ respectively. TSH range values were $1.54 \pm 1.06 \mathrm{mUI} / \mathrm{L}$ for $1 \mathrm{~T}, 1.71 \pm 0.87 \mathrm{mUI} / \mathrm{L}$ for second trimester $(2 \mathrm{~T})$ and $2.03 \pm 0.96 \mathrm{mUI} / \mathrm{L}$ for $3 \mathrm{~T}$ and FT4 range values were $0.98 \pm 0.11 \mathrm{ng} / \mathrm{dL}$ for $1 \mathrm{~T}, 0.80 \pm 0.07 \mathrm{ng} / \mathrm{dL}$ for $2 \mathrm{~T}$ and $0.79 \pm 0.08 \mathrm{ng} / \mathrm{dL}$ for $3 \mathrm{~T}$. When univariate analyses were performed, TSH concentrations in $1 \mathrm{~T}$ and $2 \mathrm{~T}$ were significantly higher in women up to 30 years of age, compared with those older than 30 . Additionally, women with low parity showed significantly higher TSH concentrations in the three trimesters in comparison with women with two or more previous pregnancies. Regarding FT4, women of 30 years of age and below showed significantly higher FT4 levels in 2T and 3T (Table 3). Additionally, FT4 was statistically lower in overweighed and obese pregnant women in comparison with women of normal weight, but the concentrations did not vary in relation with parity. The concentrations of FT4 in 1T and 2T were significantly higher in those who consumed iodised salt compared with those who did not.

Table 2: TSH values (mIU/L) in the three trimesters of gestation.

\begin{tabular}{|c|c|c|c|c|c|c|}
\hline & \multicolumn{2}{|c|}{ TSH First Trimester } & \multicolumn{2}{|c|}{ TSH Second Trimester } & \multicolumn{2}{|c|}{ TSH Third Trimester } \\
\hline & Mean (SD) & Difference in means ( $p$ ) & Mean (SD) & $\begin{array}{l}\text { Difference in means } \\
\text { (p) }\end{array}$ & $\begin{array}{c}\text { Mean } \\
\text { (SD) }\end{array}$ & Difference in means (p) \\
\hline All cases & $\begin{array}{c}1.54 \\
(1.06)\end{array}$ & & $1.71(0.87)$ & & $\begin{array}{c}2.03 \\
(0.96)\end{array}$ & \\
\hline \multicolumn{7}{|c|}{ Maternal Age } \\
\hline$\leq 30$ years & $1.80(1.31)$ & \multirow{2}{*}{0.001} & $1.86(0.97)$ & \multirow{2}{*}{0.036} & $\begin{array}{c}2.16 \\
(0.99) \\
\end{array}$ & \multirow{2}{*}{0.09} \\
\hline$>30$ years & $1.37(0.82)$ & & $1.61(0.79)$ & & $\begin{array}{c}1.94 \\
(0.93) \\
\end{array}$ & \\
\hline \multicolumn{7}{|c|}{ BMI Category } \\
\hline Normal weight & $1.49(1.02)$ & \multirow{3}{*}{$0.159^{\mathrm{a}}$} & $1.69(0.87)$ & \multirow{3}{*}{$0.873^{\mathrm{a}}$} & $\begin{array}{c}2.02 \\
(1.00)\end{array}$ & \multirow{3}{*}{$0.835^{\mathrm{a}}$} \\
\hline Overweight & $1.75(1.29)$ & & $1.76(.087)$ & & $\begin{array}{c}2.08 \\
(0.97) \\
\end{array}$ & \\
\hline Obesity & $1.44(0.80)$ & & $1.68(0.87)$ & & $\begin{array}{c}1.96 \\
(0.76)\end{array}$ & \\
\hline
\end{tabular}




\begin{tabular}{|c|c|c|c|c|c|c|}
\hline \multicolumn{7}{|c|}{ Parity } \\
\hline 0 & $2.00(1.26)$ & \multirow{4}{*}{$0.001^{\mathrm{a}}$} & $1.92(0.94)$ & \multirow{4}{*}{$0.042^{\mathrm{a}}$} & $\begin{array}{c}2.24 \\
(0.93)\end{array}$ & \multirow{4}{*}{$0.010^{\mathrm{a}}$} \\
\hline 1 & $1.40(0.90)$ & & $1.65(0.85)$ & & $\begin{array}{c}2.03 \\
(1.04)\end{array}$ & \\
\hline 2 & $1.28(0.84)$ & & $1.48(0.72)$ & & $\begin{array}{c}1.90 \\
(0.85)\end{array}$ & \\
\hline$>2$ & $1.10(0.78)$ & & $1.60(0.83)$ & & $\begin{array}{c}1.57 \\
(0.82)\end{array}$ & \\
\hline \multicolumn{7}{|c|}{ Currently Smokers } \\
\hline No & $1.59(1.12)$ & \multirow{2}{*}{0.162} & $1.77(0.88)$ & \multirow{2}{*}{0.026} & $\begin{array}{c}2.08 \\
(0.99)\end{array}$ & \multirow{2}{*}{0.077} \\
\hline Yes & $1.38(0.84)$ & & $1.46(0.76)$ & & $\begin{array}{c}1.81 \\
(0.82)\end{array}$ & \\
\hline \multicolumn{7}{|c|}{ Previously Smokers } \\
\hline No & $1.56(1.08)$ & \multirow{2}{*}{0.524} & $1.79(0.87)$ & \multirow{2}{*}{0.045} & $\begin{array}{c}2.10 \\
(0.95)\end{array}$ & \multirow{2}{*}{0.158} \\
\hline Yes & $1.45(0.96)$ & & $1.48(0.88)$ & & $\begin{array}{c}1.87 \\
(0.88)\end{array}$ & \\
\hline \multicolumn{7}{|c|}{ Working Women } \\
\hline No & $1.74(1.20)$ & \multirow{2}{*}{0.111} & $1.99(0.97)$ & \multirow{2}{*}{0.016} & $\begin{array}{c}2.32 \\
(0.99)\end{array}$ & \multirow{2}{*}{0.037} \\
\hline Yes & $1.49(1.03)$ & & $1.64(0.83)$ & & $\begin{array}{c}1.97 \\
(0.95)\end{array}$ & \\
\hline \multicolumn{7}{|c|}{ Iodised Salt } \\
\hline No & $1.64(1.15)$ & \multirow{2}{*}{0.062} & $1.82(0.94)$ & \multirow{2}{*}{0.013} & $\begin{array}{c}2.06 \\
(1.00)\end{array}$ & \multirow{2}{*}{0.519} \\
\hline Yes & $1.36(0.91)$ & & $1.52(0.69)$ & & $\begin{array}{c}1.96 \\
(0.86)\end{array}$ & \\
\hline \multicolumn{7}{|c|}{ Level of Education } \\
\hline Low & $1.49(0.90)$ & \multirow{3}{*}{$0.485^{\mathrm{a}}$} & $1.70(0.94)$ & \multirow{3}{*}{$0.258^{\mathrm{a}}$} & $\begin{array}{c}2.01 \\
(0.96)\end{array}$ & \multirow{3}{*}{$0.765^{\mathrm{a}}$} \\
\hline Middle & $1.51(1.05)$ & & $1.64(0.77)$ & & $\begin{array}{c}1.99 \\
(0.97)\end{array}$ & \\
\hline High & $1.67(1.28)$ & & $1.87(0.97)$ & & $\begin{array}{c}2.10 \\
(0.95)\end{array}$ & \\
\hline \multicolumn{7}{|c|}{ Use of Supplements } \\
\hline None & $1.61(1.13)$ & \multirow{3}{*}{0.657} & $1.81(0.86)$ & \multirow{3}{*}{$0.076^{\mathrm{a}}$} & $\begin{array}{c}2.10 \\
(1.07)\end{array}$ & \multirow{3}{*}{$0.362^{\mathrm{a}}$} \\
\hline $\begin{array}{l}\text { Potassium } \\
\text { iodide }\end{array}$ & $1.56(1.08)$ & & $1.75(0.93)$ & & $\begin{array}{c}2.02 \\
(0.91)\end{array}$ & \\
\hline Multivitamins & $1.45(0.89)$ & & $1.44(0.66)$ & & $\begin{array}{c}2.05 \\
(0.97)\end{array}$ & \\
\hline \multicolumn{7}{|c|}{ UIC $(\mu \mathrm{g} / \mathrm{L})$} \\
\hline$<150$ & $1.53(1.06)$ & \multirow{2}{*}{0.528} & $1.61(0.91)$ & \multirow{2}{*}{0.163} & $\begin{array}{c}2.03 \\
(0.96)\end{array}$ & \multirow{2}{*}{0.963} \\
\hline$>150$ & $1.45(1.00)$ & & $1.78(0.84)$ & & $\begin{array}{c}2.02 \\
(0.97)\end{array}$ & \\
\hline
\end{tabular}

\section{${ }^{a}$ ANOVA test}


Table 3: FT4 values in the three trimesters of gestation.

\begin{tabular}{|c|c|c|c|c|c|c|}
\hline & \multicolumn{2}{|c|}{ FT4 First Trimester } & \multicolumn{2}{|c|}{ FT4 Second Trimester } & \multicolumn{2}{|c|}{ FT4 Third Trimester } \\
\hline & Mean (SD) & $\begin{array}{l}\text { Difference in means } \\
\text { (p) }\end{array}$ & Mean (SD) & Difference in means (p) & Mean (SD) & Difference in means (p) \\
\hline All cases & $0.98(0.11)$ & & $0.80(0.07)$ & & $0.79(0.08)$ & \\
\hline \multicolumn{7}{|c|}{ Maternal Age } \\
\hline$\leq 30$ years & $0.99(0.10)$ & 0.159 & $0.82(0.07)$ & \multirow{2}{*}{0.002} & $0.80(0.08)$ & \multirow{2}{*}{0.012} \\
\hline$>30$ years & $0.97(0.11)$ & & $0.79(0.07)$ & & $0.77(0.08)$ & \\
\hline \multicolumn{7}{|c|}{ BMI Category } \\
\hline Normal weight & $0.99(0.10)$ & \multirow{3}{*}{$0.012^{\mathrm{a}}$} & $0.82(0.07)$ & \multirow{3}{*}{$0.001^{\mathrm{a}}$} & $0.80(0.07)$ & \multirow{3}{*}{$0.008^{\mathrm{a}}$} \\
\hline Overweight & $0.98(0.14)$ & & $0.79(0.08)$ & & $0.77(0.08)$ & \\
\hline Obesity & $0.94(0.08)$ & & $0.77(0.06)$ & & $0.76(0.07)$ & \\
\hline \multicolumn{7}{|c|}{ Parity } \\
\hline 0 & $0.97(0.10)$ & \multirow{4}{*}{$0.211^{\mathrm{a}}$} & $0.81(0.07)$ & \multirow{4}{*}{$0.799^{\mathrm{a}}$} & $0.79(0.08)$ & \multirow{4}{*}{$0.539^{\mathrm{a}}$} \\
\hline 1 & $0.99(0.11)$ & & $0.80(0.07)$ & & $0.79(0.08)$ & \\
\hline 2 & $0.96(0.11)$ & & $0.80(0.08)$ & & $0.77(0.08)$ & \\
\hline$>2$ & $0.98(0.12)$ & & $0.80(0.08)$ & & $0.79(0.08)$ & \\
\hline \multicolumn{7}{|c|}{ Currently Smokers } \\
\hline No & $0.98(0.11)$ & \multirow{2}{*}{0.083} & $0.81(0.07)$ & \multirow{2}{*}{0.385} & $0.79(0.08)$ & \multirow{2}{*}{0.877} \\
\hline Yes & $0.96(0.09)$ & & $0.80(0.08)$ & & $0.79(0.08)$ & \\
\hline \multicolumn{7}{|c|}{ Previously Smokers } \\
\hline No & $0.99(0.10)$ & \multirow{2}{*}{0.002} & $0.81(0.07)$ & \multirow{2}{*}{0.018} & $0.79(0.08)$ & \multirow{2}{*}{0.201} \\
\hline Yes & $0.94(0.09)$ & & $0.78(0.07)$ & & $0.77(0.08)$ & \\
\hline \multicolumn{7}{|c|}{ Working Women } \\
\hline No & $0.97(0.11)$ & \multirow{2}{*}{0.432} & $0.82(0.08)$ & \multirow{2}{*}{0.254} & $0.79(0.08)$ & \multirow{2}{*}{0.832} \\
\hline Yes & $0.98(0.11)$ & & $0.80(0.07)$ & & $0.79(0.08)$ & \\
\hline \multicolumn{7}{|c|}{ Iodised Salt } \\
\hline No & $0.95(0.10)$ & & $0.77(0.07)$ & & $0.75(0.07)$ & \\
\hline Yes & $1.00(0.12)$ & 0.051 & $0.80(0.08)$ & 0.010 & $0.78(0.08)$ & 0.100 \\
\hline & & & Level of & ducation & & \\
\hline Low & $0.97(0.11)$ & & $0.80(0.08)$ & & $0.78(0.08)$ & \\
\hline Middle & $0.98(0.11)$ & $0.543^{\mathrm{a}}$ & $0.80(0.07)$ & $0.450^{\mathrm{a}}$ & $0.79(0.08)$ & $0.508^{\mathrm{a}}$ \\
\hline High & $0.99(0.09)$ & & $0.81(0.06)$ & & $0.79(0.07)$ & \\
\hline & & & Use of $\mathrm{Su}$ & plements & & \\
\hline None & $0.97(0.10)$ & & $0.80(0.08)$ & & $0.80(0.08)$ & \\
\hline $\begin{array}{l}\text { Potassium } \\
\text { iodide }\end{array}$ & $0.99(0.13)$ & $0.281^{\mathrm{a}}$ & $0.81(0.08)$ & $0.868^{\mathrm{a}}$ & $0.78(0.08)$ & $0.330^{\mathrm{a}}$ \\
\hline Multivitamins & $1.00(0.11)$ & & $0.81(0.07)$ & & $0.79(0.08)$ & \\
\hline & & & UIC & $\mathrm{g} / \mathrm{L})$ & & \\
\hline$<150$ & $0.99(0.12)$ & & $0.80(0.07)$ & & $0.79(0.08)$ & \\
\hline$>150$ & $0.97(0.09)$ & 0.360 & $0.81(0.07)$ & 0.417 & $0.79(0.08)$ & 0.936 \\
\hline
\end{tabular}

\section{${ }^{\text {aANOVA test }}$}

Citation: Inés Velasco, Berta Soldevila, Raquel MartínezMondejar, Carla Muñoz, Laura Ferrer, et al. Thyroid Function Variability in a Cohort of Healthy Pregnant Women: Effect of BMI, Smoking and Iodized Salt Consumption. LOJ Phar \& Cli Res 2(2)- 2020. LOJPCR.MS.ID.000134. DOI: 10.32474/LOJPCR.2020.02.000134. 


\section{Thyroid autoimmunity}

The frequency of pregnant women positive for anti-TPOAbs was $10.6 \%, 7.8 \%$ and $7 \%$ in the $1 \mathrm{~T}, 2 \mathrm{~T}$ and $3 \mathrm{~T}$ respectively. The prevalence of anti-TgAbs was $12.2 \%, 10 \%$ and $6.2 \%$ at $1 \mathrm{~T}, 2 \mathrm{~T}$ and $3 \mathrm{~T}$ respectively. The Spearman's correlation coefficient between antiTPOAbs and anti-TgAbs was $0.47 ; 0.45$ and 0.42 in the $1 \mathrm{~T}, 2 \mathrm{~T}$ and $3 \mathrm{~T}$ respectively $(\mathrm{p}<0.001$ in all the cases). The TSH concentration at each trimester was significantly higher in those pregnant women who were anti-TPOAbs positive, but these differences were not significant in case of anti-TgAbs positive (Table 4). The FT4 levels in the $1 \mathrm{~T}$ were lower in women with positive anti-TPOAbs. (Table 4). TSH and FT4 concentrations in the three trimesters of gestation according to thyroid immune status.

Table 4: TSH and FT4 concentrations in the three trimesters of gestation according to thyroid immune status.

\begin{tabular}{|c|c|c|c|}
\hline & $\begin{array}{l}\text { Anti-TPO } \\
\text { Negative }\end{array}$ & $\begin{array}{l}\text { Anti-TPO } \\
\text { Positive }\end{array}$ & $\mathbf{p}$ \\
\hline & \multicolumn{2}{|c|}{ Mean (SD) } & \\
\hline $\begin{array}{l}\text { TSH in first } \\
\text { trimester }\end{array}$ & $1.46(0.96)$ & $2.22(1.62)$ & 0.014 \\
\hline $\begin{array}{l}\text { TSH in second } \\
\text { trimester }\end{array}$ & $1.66(0.80)$ & $2.36(1.28)$ & 0.034 \\
\hline \multirow[t]{2}{*}{$\begin{array}{l}\text { TSH in third } \\
\text { trimester }\end{array}$} & $1.98(0.92)$ & $2.84(1.27)$ & 0.016 \\
\hline & \multicolumn{2}{|c|}{ Mean (SD) } & \\
\hline $\begin{array}{l}\text { FT4 in first } \\
\text { trimester }\end{array}$ & $0.98(0.11)$ & $0.94(0.10)$ & 0.026 \\
\hline $\begin{array}{l}\text { FT4 in second } \\
\text { trimester }\end{array}$ & $0.81(0.07)$ & $0.80(0.06)$ & 0.898 \\
\hline \multirow[t]{3}{*}{$\begin{array}{l}\text { FT4 in third } \\
\text { trimester }\end{array}$} & $0.79(0.08)$ & $0.78(0.06)$ & 0.636 \\
\hline & Anti-Tg Negative & Anti-Tg Positive & \\
\hline & \multicolumn{2}{|c|}{ Mean (SD) } & \\
\hline $\begin{array}{l}\text { TSH in first } \\
\text { trimester }\end{array}$ & $1.49(0.97)$ & $1.92(1.56)$ & 0.112 \\
\hline $\begin{array}{l}\text { TSH in second } \\
\text { trimester }\end{array}$ & $1.70(0.85)$ & $1.83(1.02)$ & 0.494 \\
\hline $\begin{array}{l}\text { TSH in third } \\
\text { trimester }\end{array}$ & $2.00(0.94)$ & $2.53(1.31)$ & 0.05 \\
\hline
\end{tabular}

\begin{tabular}{|c|c|c|c|}
\hline & \multicolumn{2}{|c|}{ Mean (SD) } & \\
\hline $\begin{array}{c}\text { FT4 in first } \\
\text { trimester }\end{array}$ & $0.98(0.11)$ & $0.96(0.10)$ & 0.184 \\
\hline $\begin{array}{c}\text { FT4 in second } \\
\text { trimester }\end{array}$ & $0.81(0.07)$ & $0.81(0.06)$ & 0.99 \\
\hline $\begin{array}{c}\text { FT4 in third } \\
\text { trimester }\end{array}$ & $0.79(0.08)$ & $0.78(0.05)$ & 0.729 \\
\hline
\end{tabular}

\section{$\beta$-hCG in the first trimester}

Beta-hCG levels were significantly lower in overweighed and obese pregnant women $(152337.47 \pm 78237.95 \mathrm{mIU} / \mathrm{mL}$ in normal weighed women; $139169.68 \pm 121041.64 \mathrm{mIU} / \mathrm{mL}$ in overweighed and $102129.23 \pm 46361.49 \mathrm{mIU} / \mathrm{mL}$ in obese women, respectively; $\mathrm{p}=0.005$ ). No correlation was found between maternal age and $\beta$-hCG, but a negative correlation between pregestational BMI and $\beta$-hCG was found ( $r=-0.205 ; \mathrm{p}=0.001)$. When the sample was split out according to BMI categories, there was a strong negative correlation between maternal age and $\beta$-hCG in overweighed women $(\mathrm{r}=-0.438 ; \mathrm{p}<0.001)$.

Pregnant women who had smoked before gestation showed lower $\beta$-hCG levels compared with those who did not smoke $(122112.47 \pm 110104.65 \mathrm{mIU} / \mathrm{mL}$ vs. $150427.48 \pm 74808.07 \mathrm{mIU} /$ $\mathrm{mL}$ respectively; $\mathrm{p}=0.014$ ) and was greater in case of smoking during the pregnancy $(100365.15 \pm 53551.61 \mathrm{mIU} / \mathrm{mL}$ vs. $153156.03 \pm 93571.64 \mathrm{mIU} / \mathrm{mL}$ respectively, $\mathrm{p}<0.001)$.

\section{Maternal age}

Correlations between thyroid function and maternal age, according to different clinical conditions are shown in Table 5. TSH concentrations in $1 \mathrm{~T}$ and $2 \mathrm{~T}$ were significantly higher in women up to 30 years than in those above $30(1.80 \pm 1.30 \mathrm{mIU} /$ dl vs, $1.37 \pm 0.82 \mathrm{mIU} / \mathrm{dl}$ in $1 \mathrm{~T} ; \mathrm{p}=0.001$ and $1.86 \pm 0.97 \mathrm{mIU} / \mathrm{dl}$ vs. $1.61 \pm 0.79 \mathrm{mIU} / \mathrm{dl}$ in $2 \mathrm{~T}$; $\mathrm{p}=0.036$ ). Maternal age showed a negative correlation with TSH concentrations in $1 \mathrm{~T}$ and 2T. FT4 concentrations were significantly higher in young women (up to 30) when compared to those older than 30 years old in $2 \mathrm{~T}$ and $3 \mathrm{~T}$ $(0.99 \pm 0.10 \mathrm{ng} / \mathrm{dl}$ vs. $0.97 \pm 0.11 \mathrm{ng} / \mathrm{dl}$ for $1 \mathrm{~T}, \mathrm{p}=0.159 ; 0.82 \pm 0.07 \mathrm{ng} /$ dl vs. $0.79 \pm 0.72 \mathrm{ng} / \mathrm{dl}$ in $2 \mathrm{~T}, \mathrm{p}=0.002$; and $0.80 \pm 0.08 \mathrm{ng} / \mathrm{dl}$ vs. $0.78 \pm 0.08 \mathrm{ng} / \mathrm{dl}$ in $3 \mathrm{~T}, \mathrm{p}=0.012$ ).

Table 5: Correlation TSH and FT4 concentrations and Maternal Age, according to different clinical conditions.

\begin{tabular}{|c|c|c|c|c|c|c|}
\hline & \multicolumn{2}{|c|}{ TSH first trimester } & \multicolumn{2}{|c|}{ TSH second trimester } & \multicolumn{2}{|c|}{ TSH third trimester } \\
\hline & $\mathbf{r}$ & p & $\mathbf{r}$ & $\mathbf{p}$ & $\mathbf{r}$ & p \\
\hline All cases & -0.19 & 0.001 & -0.17 & 0.01 & -0.12 & 0.068 \\
\hline \multicolumn{7}{|c|}{ BMI Category } \\
\hline Normal weight & -0.22 & 0.003 & -0.11 & 0.195 & -0.05 & 0.593 \\
\hline Overweight & -0.26 & 0.024 & -0.46 & 0.001 & -0.36 & 0.005 \\
\hline Obesity & 0.02 & 0.908 & -0.04 & 0.821 & -0.04 & 0.81 \\
\hline
\end{tabular}




\begin{tabular}{|c|c|c|c|c|c|c|}
\hline \multicolumn{7}{|c|}{ Parity } \\
\hline 0 & -0.2 & 0.042 & -0.16 & 0.165 & -0.02 & 0.831 \\
\hline 1 & -0.15 & 0.113 & -0.2 & 0.068 & -0.2 & 0.086 \\
\hline 2 & -0.05 & 0.698 & -0.06 & 0.695 & -0.01 & 0.969 \\
\hline$>2$ & -0.21 & 0.212 & -0.09 & 0.625 & -0.03 & 0.871 \\
\hline \multicolumn{7}{|c|}{ Anti-TPO Antibodies } \\
\hline Negative & -0.21 & 0.001 & -0.18 & 0.01 & -0.16 & 0.02 \\
\hline Positive & -0.26 & 0.149 & -0.31 & 0.214 & -0.16 & 0.566 \\
\hline \multicolumn{7}{|c|}{ Anti-TgAntibodies } \\
\hline Negative & -0.23 & 0.001 & -0.21 & 0.002 & -0.18 & 0.01 \\
\hline Positive & -0.18 & 0.3 & -0.13 & 0.567 & -0.05 & 0.858 \\
\hline \multicolumn{7}{|c|}{ Currently Smokers } \\
\hline No & -0.21 & 0.001 & -0.2 & 0.005 & -0.15 & 0.042 \\
\hline Yes & -0.09 & 0.473 & 0 & 0.998 & -0.01 & 0.959 \\
\hline \multicolumn{7}{|c|}{ Previously Smokers } \\
\hline No & -0.24 & 0.001 & -0.22 & 0.004 & -0.19 & 0.015 \\
\hline Yes & -0.1 & 0.334 & -0.08 & 0.523 & 0 & 0.984 \\
\hline \multicolumn{7}{|l|}{ Working Women } \\
\hline No & -0.11 & 0.426 & -0.2 & 0.201 & -0.15 & 0.383 \\
\hline Yes & -0.21 & 0.001 & -0.11 & 0.151 & -0.07 & 0.322 \\
\hline \multicolumn{7}{|l|}{ Iodised Salt } \\
\hline No & -0.23 & 0.002 & -0.17 & 0.048 & -0.13 & 0.131 \\
\hline Yes & -0.07 & 0.57 & -0.08 & 0.547 & -0.01 & 0.942 \\
\hline \multicolumn{7}{|l|}{$\mathrm{UIC}(\mu \mathrm{g} / \mathrm{L})$} \\
\hline$<150$ & $-0,229$ & 0.006 & $-0,039$ & 0,742 & -110 & 0,332 \\
\hline \multirow[t]{3}{*}{$>150$} & & & & & $-0,188$ & 0,040 \\
\hline & \multicolumn{2}{|c|}{ FT4 first trimester } & \multicolumn{2}{|c|}{ FT4 second trimester } & \multicolumn{2}{|c|}{ FT4 third trimester } \\
\hline & $\mathbf{r}$ & p & $\mathbf{r}$ & $\mathbf{p}$ & $\mathbf{r}$ & $\mathbf{p}$ \\
\hline All cases & -0.07 & 0.225 & -0.18 & 0.006 & -0.19 & 0.004 \\
\hline \multicolumn{7}{|c|}{ BMI Category } \\
\hline Normal weight & 0 & 0.954 & -0.08 & 0.367 & -0.16 & 0.059 \\
\hline Overweight & -0.14 & 0.24 & -0.3 & 0.025 & -0.18 & 0.155 \\
\hline Obesity & -0.38 & 0.008 & -0.5 & 0.002 & -0.46 & 0.008 \\
\hline \multicolumn{7}{|c|}{ Parity } \\
\hline 0 & -0.08 & 0.441 & -0.1 & 0.387 & -0.23 & 0.035 \\
\hline 1 & 0.03 & 0.762 & -0.13 & 0.244 & -0.12 & 0.307 \\
\hline 2 & -0.14 & 0.32 & -0.25 & 0.127 & -0.19 & 0.213 \\
\hline$>2$ & -0.27 & 0.081 & -0.31 & 0.084 & -0.19 & 0.337 \\
\hline \multicolumn{7}{|c|}{ Anti-TPO Antibodies } \\
\hline Negative & -0.08 & 0.184 & -0.19 & 0.005 & -0.14 & 0.045 \\
\hline Positive & -0.09 & 0.962 & -0.23 & 0.357 & -0.32 & 0.228 \\
\hline \multicolumn{7}{|c|}{ Anti-TgAntibodies } \\
\hline Negative & -0.08 & 0.204 & -0.2 & 0.004 & -0.16 & 0.021 \\
\hline Positive & -0.03 & 0.867 & -0.76 & 0.731 & -0.04 & 0.893 \\
\hline \multicolumn{7}{|c|}{ Currently Smokers } \\
\hline No & -0.04 & 0.605 & -0.16 & 0.044 & -0.11 & 0.15 \\
\hline Yes & -0.14 & 0.175 & -0.24 & 0.047 & -0.33 & 0.004 \\
\hline
\end{tabular}




\begin{tabular}{|c|c|c|c|c|c|c|}
\hline \multicolumn{7}{|c|}{ Previously Smokers } \\
\hline No & -0.03 & 0.695 & -0.14 & 0.066 & -0.15 & 0.044 \\
\hline Yes & -0.23 & 0.055 & -0.34 & 0.019 & -0.34 & 0.018 \\
\hline \multicolumn{7}{|c|}{ Working Women } \\
\hline No & -0.32 & 0.013 & -0.49 & 0.001 & -0.55 & 0.001 \\
\hline Yes & 0 & 0.982 & -0.06 & 0.411 & -0.1 & 0.18 \\
\hline \multicolumn{7}{|c|}{ Iodised Salt } \\
\hline No & -0.07 & 0.328 & -0.16 & 0.055 & -0.21 & 0.014 \\
\hline Yes & -0.1 & 0.418 & -0.18 & 0.159 & -0.17 & 0.286 \\
\hline \multicolumn{7}{|c|}{ UIC $(\mu \mathrm{g} / \mathrm{L})$} \\
\hline$<150$ & $-0,006$ & 0,940 & $-0,139$ & 0,238 & $-0,157$ & 0,165 \\
\hline$>150$ & $-0,135$ & 0,191 & $-0,189$ & 0,032 & $-0,183$ & 0,046 \\
\hline
\end{tabular}

\section{Pregestational body mass index (BMI)}

BMI showed a negative correlation with FT4 in the three trimesters of gestation (Table 5). This negative correlation was significant in women above 30 years old and in women who did not smoke neither during nor before pregnancy. FT4 concentrations were significantly higher in young women (up to 30) when compared to those older than 30 years old, and this difference increased particularly in the first trimester in the case of obese women $\left(\mathrm{BMI} \geq 30 \mathrm{~kg} / \mathrm{m}^{2}\right)(0.97 \pm 0.07 \mathrm{ng} / \mathrm{dl}$ vs $0.90 \pm 0.07 \mathrm{ng} / \mathrm{dl}$ for the first trimester, $\mathrm{p}=0.003 ; 0.80 \pm 0.05 \mathrm{ng} / \mathrm{dl}$ vs $0.75 \pm 0.06 \mathrm{ng} / \mathrm{dl}$ in the second trimester, $\mathrm{p}=0.011$; and $0.79 \pm 0.07 \mathrm{ng} / \mathrm{dl}$ vs $0.73 \pm 0.07 \mathrm{ng} / \mathrm{dl}$ in the third trimester, $\mathrm{p}=0.035$ ). Besides, the negative correlation between maternal age and FT4 became stronger in obese women (Table 4).

\section{Multiple linear regression of thyroid function parameters}

We searched for maternal variables that may explain the variability of TSH and FT4 within normal reference ranges in each trimester of pregnancy, designing multiple linear regression models for each parameter (TSH, FT4) in the three trimesters. The best model for TSH at $1 \mathrm{~T}$ included FT4 at $1 \mathrm{~T}(\mathrm{p}<0.001)$, parity $(\mathrm{p}<0.001)$, anti-TPOAbs $(\mathrm{p}<0.001)$, gestational age $(\mathrm{p}=0.008)$, $\beta$-hCG $(p=0.014)$, and current smoking habit $(p=0.020)$, with $\mathrm{R}^{2}=0.240 ; \mathrm{p}<0.001$. For TSH in $2 \mathrm{~T}$, the predictive variables were TSH at $1 \mathrm{~T}(\mathrm{p}<0.001), \beta$-hCG $(\mathrm{p}<0.001)$, gestational age $(\mathrm{p}=0.001)$, and parity $(\mathrm{p}=0.06)$, with $\mathrm{R}^{2}=0.621 ; \mathrm{p}<0.001$. And for TSH in $3 \mathrm{~T}$, the predictive variables were TSH at $2 \mathrm{~T}(\mathrm{p}<0.001)$ and TSH at $1 \mathrm{~T}$ $(\mathrm{p}=0.003)$ and parity $(\mathrm{p}=0.049)$, with $\mathrm{R}^{2}=0.685 ; \mathrm{p}<0.001$. For FT4, the best model at 1 T included TSH at $1 \mathrm{~T}(\mathrm{p}<0.001), \beta-\mathrm{hCG}(\mathrm{p}=0.005)$, pregestational BMI $(p=0.005)$ and maternal age $(p=0.027)$ with $\mathrm{R}^{2}=0.151 ; \mathrm{p}<0.001$. For FT4 in $2 \mathrm{~T}$, the predictive variables were FT4 in $1 \mathrm{~T}(\mathrm{p}<0.001)$, TSH in $2 \mathrm{~T}(\mathrm{p}=0.005)$, pregestational BMI $(\mathrm{p}=0.007)$ and maternal age $(\mathrm{p}=0.024)$, with $\mathrm{R}^{2}=0.356$; $\mathrm{p}<0.001$. And for FT4 in $3 \mathrm{~T}$, the predictive variables were FT4 at $2 \mathrm{~T}(\mathrm{p}<0.001)$ and FT4 at $1 \mathrm{~T}$ trimester $(\mathrm{p}=0.001)$ and pregestational $\mathrm{BMI}(\mathrm{p}=0.021)$ with $\mathrm{R}^{2}=0.550 ; \mathrm{p}<0.001$.

\section{Discussion}

Our study shows the complex interplay between maternal age, pregestational BMI, parity and smoking habit as modulators of thyroid function variability in healthy pregnant women. In our cohort, univariate analyses identified differences in 1T FT4 levels according to BMI, smoking habit and $\beta$-hCG. We also found that maternal age negatively correlated with TSH concentrations and this relationship was influenced by thyroid autoimmunity, smoking habit and the intake of iodised salt. The variations of TSH concentrations in $1 \mathrm{~T}$, and to a lesser extent in the $2 \mathrm{~T}$ and $3 \mathrm{~T}$, were also related to $\beta$-hCG. In addition, women older than 30 showed lower concentrations of TSH in $1 \mathrm{~T}$ and $2 \mathrm{~T}$ compared to those under 30 , thus accounting for a negative correlation between TSH and maternal age. Moreover, FT4 was significantly lower in women of 30 and above, with a statistically significant negative correlation. Pregnant women older than 30 show lower TSH concentrations for a given FT4 value, which may indicate a less effective direct stimulatory action of $\beta$-hCG, as if some decreased sensitivity of maternal thyroid gland to $\beta$-hCG was ongoing with age. However, TSH concentrations were also significantly lower in women with high parity in our sample and the negative correlation between TSH and maternal age did not persist when the sample was stratified according to parity, rendering maternal age as a likely confounder. Parity $>2$, but not maternal age, has been associated with lower thyroidal response to $\beta$-hCG in other studies $[11,14]$. Some studies have thoroughly analysed those variables with substantial effect on serum $\beta$-hCG levels during pregnancy, such as gestational age at assessment, maternal age, weight and cigarette smoking [18] and their relation to thyroid function tests. We found a strong negative effect of BMI and smoking habit on $\beta$-hCG concentration in $1 \mathrm{~T}$, in consonance with other authors [11]. Since obesity has been associated with a lower thyroidal response to $\beta$-hCG stimulation [11], the low levels of FT4 that we found in women with high BMI or who had smoked before the pregnancy might be interpreted as a poor $\beta$-hCG-mediated stimulation [19]. 
TSH seems to be positively related to the degree of obesity [20], meanwhile fat accumulation has been associated with lower FT4[21]. In our study, the negative effect of BMI on FT4 levels is steadily present in the three trimesters. It has been suggested that women with abdominal obesity show a high conversion of T4 to T3 due to increased deiodinase activity, as a compensatory mechanism to improve energy expenditure [22]. Additionally, the presence of anti-TPOAbs was associated with higher levels of TSH in the three trimesters of gestation and a lower level of FT4 in the 1T, while anti-TgAbs did not affect TSH nor FT4 concentrations. These results reinforce the importance of anti-TPOAbs-positive as a marker of impaired thyroid response to $\beta$-hCG stimulation $[11,6]$.

There is solid evidence that current smokers have significantly lower serum TSH levels than non-smokers, probably in relation with leptin levels [23]. This effect is dose-dependent, disappears slowly after smoking cessation and is not associated with iodine intake [24]. Our results show a difference in mean TSH levels in 2T greater in current smokers than in stopped smokers. However, this difference did not reach statistical significance for TSH in 1T nor in $3 \mathrm{~T}$, which might be due to the interaction with potential confounders such as BMI or parity [25]. Finally, pregnant women who consumed iodised salt showed higher levels of FT4 in 1T and 2T and did not show the negative effect of BMI or maternal age on FT4, particularly in the $1 \mathrm{~T}$ of pregnancy. Long-term iodized salt consumption before pregnancy significantly improves maternal thyroid economy and reduces the risk of maternal thyroid insufficiency during gestation, probably because of optimized intrathyroidal iodine stores [26]. A prior study of our group found that iodised salt (consumed at least one year before becoming pregnant) may be as effective as other forms of iodoprophylaxis in terms of infant neurocognitive development [27-29]. Our study was focused on healthy pregnant women, and even the obese or smoker participants had no other comorbidities to be considered at risk to develop any obstetric or perinatal complication. Maternal thyroid function in the first trimester can be useful for predicting thyroid function throughout the pregnancy [30], together with BMI and smoking habit as modulating factors. Therefore, preconceptional identification and modification of preventable maternal conditions that could potentially undermine thyroid function -such as those latter BMI, smoking-, especially at early stages of pregnancy is important.

\section{Funding Sources}

This study has been funded by Instituto de Salud Carlos III through the projects PI15/02192; PI15/02200 and Pi18/01857 (Co-funded by European Regional Development Fund "A way to make Europe").

\section{Disclosure Statement}

None of the authors have any conflicts of interest to declare.

\section{References}

1. Forhead AJ, Fowden AL (2014) Thyroid hormones in fetal growth and prepartum maturation. J Endocrinol 221(3): R87-R103.

2. Männistö T, Mendola P, Grewal J, Xie Y, Chen Z, et al. (2013) Thyroid diseases and adverse pregnancy outcomes in a contemporary US cohort. The Journal of Clinical Endocrinology Metabolism 98(7): 2725-2733.

3. Maraka S, Ospina NM, O’Keeffe DT, Espinosa De Ycaza AE, Gionfriddo MR, et al. (2016) Subclinical Hypothyroidism in Pregnancy: A Systematic Review and Meta Analysis.Thyroid 26(4):580-590.

4. Obregon MJ, Calvo RM, Escobar Del Rey F, Morreale De Escobar G (2007) Ontogenesis of thyroid function and interactions with maternal function. Endocr Dev 10:86-98.

5. Rovet JF (2014) The role of thyroid hormones for brain development and cognitive function. Endocr Dev 26:26-43.

6. Moleti M, Trimarchi F, Vermiglio F (2014) Thyroid physiology in pregnancy. Endocr Pract 20(6): 589-596.

7. Licht P, Russu V, Wildt L (2001) On the role of human chorionic gonadotropin ( $\mathrm{hCG}$ ) in the embryo-endometrial microenvironment: implications for differentiation and implantation. Semin Reprod Med 19(1): 37-47.

8. Alexander EK, Pearce EN, Brent GA, Brown RS, Chen H, et al. (2017) 2017 Guidelines of the American Thyroid Association for the Diagnosis and Management of Thyroid Disease During Pregnancy and the Postpartum. Thyroid 27(3): 315-389.

9. Glinoer D (1997) The regulation of thyroid function in pregnancy: pathways of endocrine adaptation from physiology to pathology. Endocr Rev 18(3): 404-33.

10. Fraenkel M, Shafat T, Cahn A, Erez O, Novack V, et al. (2018) Low thyroidstimulating hormone and its persistence beyond the first trimester of pregnancy. Int J Gynaecol Obstet. 142(3): 270-276.

11. Haddow JE, McClain MR, Lambert-Messerlian G, Palomaki GE, Canick JA, et al. (2008) Variability in thyroid-stimulating hormone suppression by human chorionic [corrected] gonadotropin during early pregnancy. J Clin Endocrinol Metab 93(9): 3341-3347.

12. Korevaar TI, Steegers EA, Pop VJ, Broeren MA, Chaker L, et al. (2017) Thyroid Autoimmunity Impairs the Thyroidal Response to Human Chorionic Gonadotropin: Two Population-Based Prospective Cohort Studies. J Clin Endocrinol Metab 102(1): 69-77.

13. Wang X, Sun X, Yang L, Tang R, Zhou J, et al. (2019) Maternal thyroidstimulating hormone level in the first trimester and sex ratio at birth. Endocr Pract 25(4):315-319.

14. Korevaar TI, Rijke YB De, Chaker L, Medici M, Jaddoe VW, et al. (2017) Stimulation of Thyroid Function by Human Chorionic Gonadotropin During Pregnancy: A Risk Factor for Thyroid Disease and a Mechanism for Known Risk Factors. Thyroid 27(3): 440-450.

15. Moleti M, Di Bella B, Giorgianni G, Mancuso A, De Vivo A, et al. (2011) Maternal thyroid function in different conditions of iodine nutrition in pregnant women exposed to mild-moderate iodine deficiency: an observational study. Clinical Endocrinology 74(6):762-768.

16. Pearce EN, Oken E, Gillman MW, Lee SL, Magnani B, et al. (2008) Association of first-trimester thyroid function test values with thyroperoxidase antibody status, smoking, and multivitamin use. Endocr Pract 14(1): 33-39.

17. Veltri F, Poppe K (2018) Variables Contributing to Thyroid (Dys)Function in Pregnant Women: More than Thyroid Antibodies? Eur Thyroid J 7(3): 120-128.

18. Wright D, Papadopoulos S, Silva M, Wright A, Nicolaides KH (2015) Serum free $\beta$-human chorionic gonadotropin in the three trimesters 
of pregnancy: effects of maternal characteristics and medical history Ultrasound Obstet Gynecol 46(1): 51-59.

19. Mouzon SH, Lassance L (2015) Endocrine and metabolic adaptations to pregnancy; impact of obesity. Horm Mol Biol Clin Investig 24(1): 65-72.

20. Fontenelle LC, Feitosa MM, Severo JS, Freitas TE, Morais JB, et al. (2016) Thyroid Function in Human Obesity: Underlying Mechanisms. Horm Metab Res 48(12): 787-794.

21. Biondi B (2010) Thyroid and obesity: an intriguing relationship. The Journal of Clinical Endocrinology Metabolism 95(8): 3614-3617.

22. De Pergola G, Ciampolillo A, Paolotti S, Trerotoli P, Giorgino R (2007) Free triiodothyronine and thyroid stimulating hormone are directly associated with waist circumference, independently of insulin resistance, metabolic parameters and blood pressure in overweight and obese women. Clin Endocrinol (Oxf) 67(2): 265-269.

23. Lucas A, Granada ML, Olaizola I, Castell C, Julián MT, et al. (2013) Leptin and thyrotropin relationship is modulated by smoking status in euthyroid subjects. Thyroid 23(8): 964-970.

24. Wiersinga WM (2013) Smoking and thyroid. Clin Endocrinol (Oxf) $79(2): 145-151$.
25. Asvold BO, Bjøro T, Nilsen TI, Vatten LJ (2008) Tobacco smoking and thyroid function: is weight gain a confounder? Arch Intern Med 168(1): 110-114.

26. Moleti M, Lo Presti VP, Campolo MC, Mattina F, Galletti M, (2008) Iodine prophylaxis using iodized salt and risk of maternal thyroid failure in conditions of mild iodine deficiency. J Clin Endocrinol Metab 93(7): 2616-2621.

27. Santiago P, Velasco I, Muela JA, Sánchez B, Martínez J, et al. (2013) Infant neurocognitive development is independent of the use of iodised salt or iodine supplements given during pregnancy. Br J Nutr 110(5): 831-839.

28. Rebagliato M, Murcia M, Espada M, Alvarez Pedrerol M, Bolúmar F (2010) Iodine intake and maternal thyroid function during pregnancy. Epidemiology 21(1): 62-69.

29. Velasco I, Gónzalez Romero S, Soriguer F (2010) Iodine and thyroid function during pregnancy. Epidemiology 21(3):428-429.

30.Pop V, Broeren M, Wijnen H, Endendijk J, Van Baar A, et al. (2018) Longitudinal Trajectories of Gestational Thyroid Function: A New Approach to Better Understand Changes in Thyroid Function. J Clin Endocrinol Metab 103(8): 2889-2900.

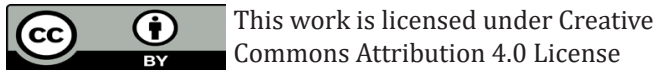

To Submit Your Article Click Here:

Submit Article

DOI: $10.32474 /$ LOJPCR.2020.02.000134

Lupine Online Journal of
Pharmacology \& Clinical Research
Assets of Publishing with us
- Global archiving of articles
- Immediate, unrestricted online access
- Rigorous Peer Review Process
Pharmaneology \& E Clinical Research

